\title{
Enforcing shaping of thin gel sheets by anisotropic swelling
}

\author{
Daniele Battista $^{\mathrm{a}}$, Michele Curatolo ${ }^{\mathrm{a}}$, Paola Nardinocchi ${ }^{\mathrm{a}, *}$ \\ ${ }^{a}$ Dipartimento di Ingegneria Strutturale e Geotecnica \\ Sapienza Università di Roma, I-00184 Roma, Italy
}

\begin{abstract}
This paper investigates swelling-induced shaping in bilayered thin plates. Sphere-like and nearly developable shapes are realized and the ability to control a specific shaping, shifting from one shape to another, under anisotropic swelling is investigated. It is shown that reinforcing fibers can be crucial in controlling shaping under swelling and dramatically affect the characteristics of the final shapes.
\end{abstract}

Keywords: thin sheets, anisotropic swelling, shaping problems

\section{Introduction}

Swelling and shrinking of polymer gels have been widely used as driving forces for changing the shape of materials. In response to different stimuli, such as changes in temperature or $\mathrm{pH}$, the solvent uptake inside the cross-linked network can change resulting in reversible volumetric expansion or shrinkage. Most gels have a long response time, and the corresponding changes in shape are typically slow, generally due to local volume changes in the material corresponding to solvent uptake and release. Inhomogeneous swelling (shrinking) can determine dramatic changes in shape, especially in thin structures which can also quickly shift from one shape to another (see $[23,21,29,32,14,24,6,38,39,40,22,3$, and references therein for thin gel and polymeric structures). In thin shells, in-plane and through-the-thickness inhomogeneous swelling, corresponding to in-plane or through-the-thickness inhomogeneous material, drive these changes in shape and realize domes and other surfaces from plates [34, 37, 35].

Swelling in gel bodies can be viewed as an elastic growth, as it is a growth of the body due to the elastic stretching induced by solvent uptake of the polymeric network. A gel body stores elastic energy even if it freely swells from a dry state under no constraints and no loads. On the contrary, (inelastic) growth processes can determine changes in body shape without any storage of elastic energy, if growth deformations are compatible

${ }^{*}$ Corresponding Author: P. Nardinocchi, Dipartimento di Ingegneria Strutturale e Geotecnica, via Eudossiana 18, I-00184 Roma, tel: 003906 44585242, fax: 0039064884852.

Email addresses: daniele.battista@uniroma1.it (Daniele Battista),

michele.curatolo@uniroma3.it (Michele Curatolo), paola.nardinocchi@uniroma1.it (Paola

Nardinocchi) 
and realizable. So, swelling and growth are quite different phenomena from an energetic point of view, especially when they come together (i.e. in active gels [4, 2]). There is a clear distinction between them and a full modeling of their interactions is fundamentally important to accurately describe the combined processes $[8]$. Nevertheless, reduced models of growing plates and shells have often been used to study swelling in thin structures [23, 13, 25]. The reason is that, for thin structures, the growth approach to the elastic problem, based on the multiplicative decomposition of the deformation gradient, yields simplified models which allow for semi-analytical solutions. However, the threedimensional nature of the swelling processes, which are locally isotropic processes, as well as the differential capability to store energy with respect to growth processes, can deliver important differences in the realized steady and swollen configurations.

The topic of the paper is swelling-driven bending of thin plates. The strategy used to get plate bending under swelling is based on bonding one above the other two layers with different elastic properties to realize a through-the-thickness inhomogeneous swelling. The amount of bending depends on the swelling mismatch between the layers; on the other side, the final shaping depends on different geometrical and mechanical factors. The goal is to discuss the shaping of bilayered plates from the three-dimensional fully coupled stress-diffusion nonlinear model the point of view, presented in [26] and largely tested and discussed in [28, 30, 31, 9, 10, 11, 12] and to compare it with other results based on growth approaches. A large range of swelling mismatches and plates with different slenderness, aspect ratio and stiffness were selected. Bifurcation from the spherical shape is identified by aspect ratios different from 1 .

The results are also reviewed within the context of the non-euclidean plate theory [35] and discussed with reference to a revised version of the bifurcation formula produced in 33 .

Finally, it is shown how shaping strategy can be controlled by realizing bilayered plates which include anisotropic layers. In this case, reinforcing fibers can be crucial in controlling shaping under swelling and dramaticallyaffect the characteristics of the final shapes.

\section{Steady states of swelling processes}

Swelling is studied starting from the multiphysics model presented and discussed in Ref. 26 and successively refined in Refs. 28, 9, 11, 12. The key elements of the model are shortly reviewed.

Water-polymer mixture is modeled as a homogenized continuum body, allowing for a mass flux of the solvent (see also Refs. 19, 20, 42, 7). The dry-reference state $\mathcal{G}_{d}$ of the gel is a shell, identified with the region of the Euclidean space $\mathcal{E}$ it occupies. The state variables of the model are the displacement field $\mathbf{u}_{d}$ from $\mathcal{G}_{d}$ and the molar waterconcentration $c_{d}$ per unit dry volume. The two state variables satisfy the following volumetric constraint $J_{d}=\operatorname{det} \mathbf{F}_{d}=\hat{J}_{d}\left(c_{d}\right)=1+\Omega c_{d}$, implying that any changes in volume of the gel is accompanied by an equivalent uptake or release of water content. Therein, $\mathbf{F}_{d}=\mathbf{I}+\nabla \mathbf{u}_{d}$ is the deformation gradient and $\Omega$ is the molar volume of the water. 
As prescribed by the Flory-Rehner thermodynamic model [16, 17], the free energy $\psi$ per unit dry-volume depends on $\mathbf{F}_{d}$ through an elastic component $\psi_{e}$, and on $c_{d}$ through a polymer-water mixing energy $\psi_{m}: \psi=\psi_{e}+\psi_{m}$. We introduce a relaxed free-energy $\psi_{r}$, which includes the volumetric constraint, as $\psi_{r}\left(\mathbf{F}_{d}, c_{d}, p\right)=\psi_{e}\left(\mathbf{F}_{d}\right)+\psi_{m}\left(c_{d}\right)-p\left(J_{d}-\right.$ $\left.\hat{J}_{d}\left(c_{d}\right)\right)$ with the pressure $p$ as the reaction to the volumetric constraint, which maintains the volume change $J_{d}$ due to the displacement equal to the one due to solvent absorption or release $\hat{J}_{d}\left(c_{d}\right)$. The constitutive equation for the dry-reference stress $\mathbf{S}_{d}$ and for the chemical potential $\mu$ are consistently derived as

$$
\mathbf{S}_{d}=\frac{\partial \psi_{e}}{\partial \mathbf{F}_{d}}-p \mathbf{F}_{d}^{\star} \quad \text { and } \quad \mu=\frac{\partial \psi_{m}}{\partial c_{d}}+p \Omega
$$

being $\mathbf{F}_{d}^{\star}=J_{d} \mathbf{F}_{d}^{-T}$. For isotropic gels, the elastic component $\psi_{e}$ of the free energy takes a neo-Hookean form, whereas for anisotropic gels a further component has been added which accounts for the reinforcing effect of the fibres [31]. The polymer-water mixing energy $\psi_{m}$ always keeps the Flory-Huggins isotropic form. So, introduced the unit vector field e which identifies the fiber direction field in transversely isotropic gels, we have:

$$
\psi_{e}\left(\mathbf{F}_{d}\right)=\frac{G_{d}}{2}\left(\mathbf{F}_{d} \cdot \mathbf{F}_{d}-3\right) \quad \text { or } \quad \psi_{e}\left(\mathbf{F}_{d}\right)=\frac{G_{d}}{2}\left(\mathbf{F}_{d} \cdot \mathbf{F}_{d}-3\right)+\frac{G_{d} \gamma}{2}\left(\mathbf{F}_{d} \mathbf{e} \cdot \mathbf{F}_{d} \mathbf{e}-1\right)^{2},
$$

and

$$
\psi_{m}\left(c_{d}\right)=\frac{\mathcal{R} T}{\Omega} h\left(c_{d}\right) \quad \text { with } \quad h\left(c_{d}\right)=\Omega c_{d} \log \frac{\Omega c_{d}}{1+\Omega c_{d}}+\chi \frac{\Omega c_{d}}{1+\Omega c_{d}} ;
$$

where $G_{d}$ represents the shear modulus of the dry polymer, $\mathcal{R}$ represents the universal gas constant, $T$ represents the temperature and $\chi$ represents the Flory parameter. Whereas the constitutive equation for the chemical potential does not change for isotropic/anisotropic gels, and is

$$
\mu=\hat{\mu}\left(c_{d}\right)=\hat{\mu}\left(J_{d}\right)=\mathcal{R} T\left(\log \frac{J_{d}-1}{J_{d}}+\frac{1}{J_{d}}+\frac{\chi}{J_{d}^{2}}\right),
$$

the constitutive equations for the stress in the isotropic and anisotropic case are

$$
\mathbf{S}_{d}=G_{d} \mathbf{F}_{d}-p \mathbf{F}_{d}^{\star} \quad \text { or } \quad \mathbf{S}_{d}=G_{d} \mathbf{F}_{d}+2 G_{d} \gamma\left(\mathbf{F}_{d} \mathbf{e} \cdot \mathbf{F}_{d} \mathbf{e}-1\right) \mathbf{F}_{d} \mathbf{e} \otimes \mathbf{e}-p \mathbf{F}_{d}^{\star} .
$$

Typically, thermodynamical issues drive the constitutive representation of the solvent flux $\mathbf{h}_{d}$ in terms of the gradient $\nabla \mu$ of the chemical potential as $\mathbf{h}_{d}=-\mathbf{M}\left(\mathbf{F}_{d}, c_{d}\right) \nabla\left(\hat{\mu}\left(c_{d}\right)+\right.$ $p \Omega)$ with the mobility tensor $\mathbf{M}\left(\mathbf{F}_{d}, c_{d}\right)=D / \mathcal{R} T c_{d} \mathbf{C}_{d}^{-1}$ and $\mathbf{C}_{d}=\mathbf{F}_{d}^{T} \mathbf{F}_{d}$ as a positive definite tensor depending on the diffusivity $D$ of the polymer. Here, we only aim to characterize the stationary swollen states of thin bilayered structures, delivered by the equilibrium equations of the model in the form:

$$
0=\operatorname{div} \mathbf{S}_{d} \text { and } 0=-\operatorname{div} \mathbf{h}_{d} \quad \text { in } \mathcal{G}_{d} .
$$

In the present studies, they are supplemented by the boundary mechanical $\mathbf{S}_{d} \mathbf{m}=\mathbf{t}$ and chemical $\hat{\mu}\left(c_{s}\right)+p \Omega=\mu_{e}$ conditions on $\partial \mathcal{G}_{d}$, with the latter corresponding to an implicit condition on the concentration field $c_{s}$ on $\partial \mathcal{G}_{d}$ controlled by assigning the external chemical potential $\mu_{e}$.

It is worth noting that the pressure term $p$ in the constitutive equations.. 


\subsection{Swelling mismatch in bilayered structures}

Bilayered plates bend under swelling due to the mismatch between the shear moduli and/or the Flory parameters of the two plate-like parts. The issue has already been largely discussed in the literature [1, 15, 27, 34, 35], and can be described starting from the free-swelling solution of the stress-diffusion problem corresponding to a body which is embedded into a bath of assigned chemical potential $\mu_{e}$. A homogeneous body attains a swollen stress-free state with $\mathbf{S}_{d}=\mathbf{0}$ and $\mathbf{h}_{d}=0$, identified by the deformation gradient $\mathbf{F}_{d}=\lambda_{o} \mathbf{I}$ with the free-swelling stretch $\lambda_{o}$ the solution of the equilibrium equation:

$$
\mathcal{R} T\left(\log \frac{\lambda_{o}^{3}-1}{\lambda_{o}^{3}}+\frac{1}{\lambda_{o}^{3}}+\frac{\chi}{\lambda_{o}^{6}}\right)+\frac{G_{d} \Omega}{\lambda_{o}}=\mu_{e} .
$$

Equation (2.7) shows that the uniform swelling ratio $\lambda_{o}$ is completely determined by the shear modulus $G$ once the external chemical potential $\mu_{e}$ and the Flory parameter $\chi$ have been fixed.

When two bonded layers of different shear modulus swell into a homogeneous bath of assigned chemical potential, the mismatch between the uniform swelling ratios which would correspond to the two layers if each of them was free from the other, is one of the determinants of the bending intensity. It has been shown that, for $1 / \lambda_{o}^{3} \rightarrow 0$, the mismatch $\delta$ between the uniform swelling ratios $\lambda_{o t}$ and $\lambda_{o b}$ of the top and bottom layers, respectively, can be written as

$$
\delta=\frac{\lambda_{o t}}{\lambda_{o b}}=\left(\frac{G_{b}}{G_{t}}\right)^{1 / 5}=\alpha^{-1 / 5} \quad \text { with } \quad \alpha=\frac{G_{t}}{G_{b}} .
$$

When one of the two layers is unidirectionally fiber reinforced, the swelling mismatch between the two layers may be different along the fiber direction and in the transverse direction. Indeed, a homogeneous anisotropic body with fibers attains a swollen stressfree state with $\mathbf{S}_{d}=\mathbf{0}$ and $\mathbf{h}_{d}=0$, identified by the deformation gradient $\mathbf{F}_{d}=\lambda_{o \|} \mathbf{e} \otimes$ $\mathbf{e}+\lambda_{o \perp} \check{\mathbf{I}}$ being $\lambda_{o \|}$ and $\lambda_{o \perp}$ the linear swelling ratios along the fiber direction $\mathbf{e} \otimes \mathbf{e}$, and in the orthogonal plane, respectively, and $\check{\mathbf{I}}=\mathbf{I}-\mathbf{e} \otimes \mathbf{e}$. Moreover, the free-swelling stretches $\lambda_{o \|}$ and $\lambda_{o \perp}$ are solutions of the equilibrium equations:

$$
\lambda_{o \perp}^{2}=\left(1+2 \gamma\left(\lambda_{o \|}^{2}-1\right)\right) \lambda_{o \|}^{2} \quad \text { and } \quad \frac{G \Omega}{\lambda_{o \|}}=\mu_{e}-\hat{\mu}\left(J_{d}\right), \quad J_{d}=\lambda_{o \|} \lambda_{o \perp}^{2}
$$

Equation in $2.9 \lambda_{1}$ delivers a representation of $\lambda_{o \perp}^{2}$ in terms of $\lambda_{o \|}^{2}$; by using it in $\sqrt{2.9} \lambda_{2}$, we get the relation between $\lambda_{o \perp}$ and the external chemical potential $\mu_{e}$. It is worth noting that the isotropic free-swelling ratio $\lambda_{o}$ always stays between $\lambda_{o \|}$ and $\lambda_{o} \perp$ for the same values of the other parameters:

$$
\lambda_{o \|}<\lambda_{o}<\lambda_{o \perp}
$$

Hence, we can define differential swelling mismatches depending on the directions and on the position of the fibers within the top or bottom layer. For fiber reinforced top or 
bottom layers, we have

$$
\delta_{\|}^{t}=\frac{\lambda_{o t \|}}{\lambda_{o b}} \quad \text { and } \quad \delta_{\perp}^{t}=\frac{\lambda_{o t \perp}}{\lambda_{o b}} \quad \text { or } \quad \delta_{\|}^{b}=\frac{\lambda_{o t}}{\lambda_{o b \|}} \quad \text { and } \quad \delta_{\perp}^{b}=\frac{\lambda_{o t}}{\lambda_{o b \perp}} .
$$

Due to 2.10), it holds

$$
\delta_{\|}^{t}=\frac{\lambda_{o t \|}}{\lambda_{o b}}<\frac{\lambda_{o t}}{\lambda_{o b}}=\delta \quad \text { and } \quad \delta_{\perp}^{t}=\frac{\lambda_{o t \perp}}{\lambda_{o b}}>\frac{\lambda_{o t}}{\lambda_{o b}}=\delta,
$$

and

$$
\delta_{\|}^{b}=\frac{\lambda_{o t}}{\lambda_{o b \|}}>\frac{\lambda_{o t}}{\lambda_{o b}}=\delta \quad \text { and } \quad \delta_{\perp}^{b}=\frac{\lambda_{o t}}{\lambda_{o b \perp}}>\frac{\lambda_{o t}}{\lambda_{o b}}=\delta,
$$

Specifically, if the elastic moduli of the two layers are the same, we have that $\delta=$ $\delta_{\|}^{b} \delta_{\|}^{t}=\delta_{\perp}^{b} \delta_{\perp}^{t}$. As shown in Section 4 , the shaping problems, when anisotropic layers are included in the assembled structure, caused by the differential swelling mismatches raise some interesting questions.

\section{Isotropic swelling of bilayer thin sheets}

Realizing thin multilayered plates by bonding at least two layers with different elastic moduli, and with at least one of the two as a stimuli-responsive layer is a strategy to get plate bending in absence of mechanical actions. Under the stimulus, the mismatched response of the two layers delivers a strain gradient along the thickness which allows to realize bending in absence of mechanical actions.

We consider bilayered isotropic plates of thickness $h$ and sides $L_{1}$ and $L_{2}$, with $h / L_{\eta}<<$ $1(\eta=1,2)$. We assume the ratio $\beta$ between the thickness $h_{t}$ of the top layer and the thickness $h$ of the plate fixed. Once embedded into a solvent bath, each layer swells as much as its shear modulus and the bond with the other layer allows. The plate increases its thickness and, due to the swelling mismatch $\delta$ between the two layers, bends.

The problem is set within the stress-diffusion model illustrated in the Section 2 and the isotropic elastic energy is chosen in the form 2.3$)_{1}$. We are interested in the equilibrium solution of the problem and solve it by using Stationary Solutor in the finite element software COMSOL Multiphysics. We use a mesh composed of parallelepiped cells with at least six elements for each direction and the stationary solver with the Newton nonlinear method to solve all the equations of the problem in a weak form. High orders shape functions are also used; specifically, we use quintic order Lagrange shape functions for the balance of forces and the balance of solvent mass and a quartic discontinous Lagrange shape function for the Lagrangian multiplier of the volumetric constraint equation. Finally, the convergence is verified by testing different sizes of mesh and by increasing the order of the shape functions.

\subsection{From flat to double-curve shapes}

We started our analyses by implementing a wide range of swelling tests keeping fixed the aspect ratio $\mathrm{AR}=1$ of the plate, that is, assuming $L_{1}=L_{2}=L($ and $L=1 \mathrm{~cm}$ ), 
and changing both the slenderness $h / L$, in the interval $(0.08,0.2)$, and the ratio $\alpha$ in the interval $(0.3,1)$. We also fix $\beta=0.6^{1}$. At each $\alpha$, it corresponds a swelling mismatch $\delta=\lambda_{o t} / \lambda_{o b}$, due to the equation (2.11). In our tests, $\delta$ varies in the interval $(1.3,1)$, determining a maximum differential strain $\varepsilon_{o t}-\varepsilon_{o b} \simeq 0.3$. It is worth noting that the same swelling mismatch holds when the bottom layer (and, as a consequence assuming $\alpha$ as fixed, the top layer) is softer and softer.
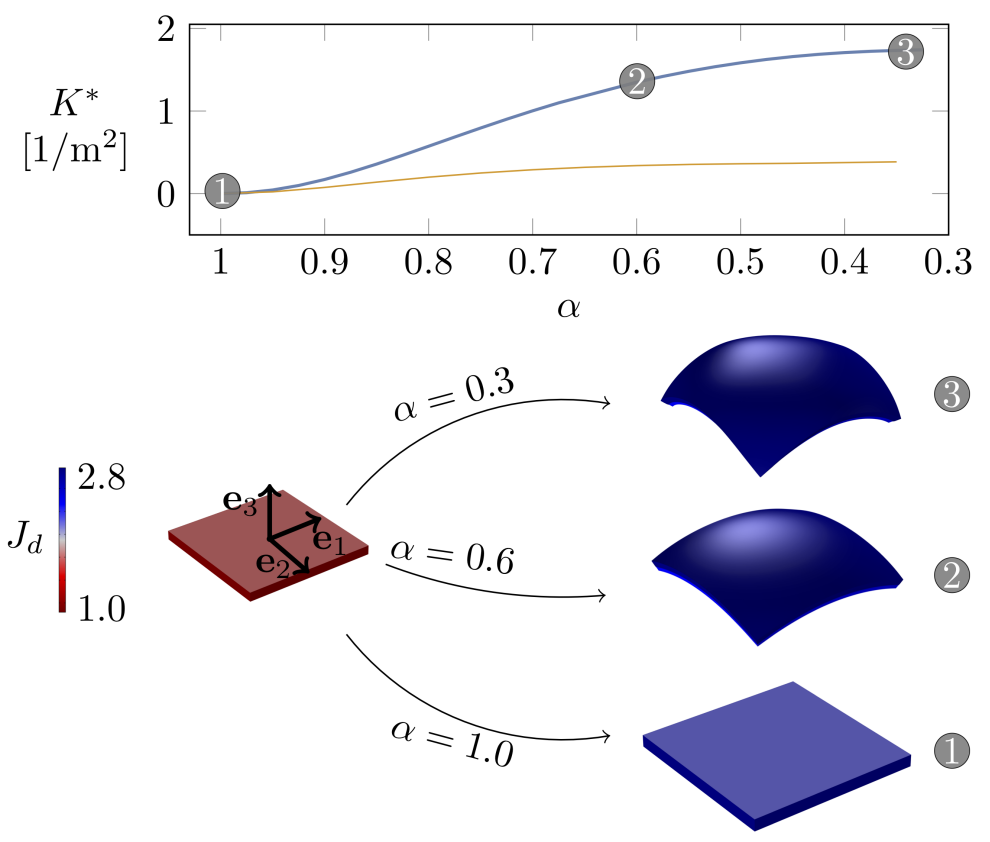

Figure 1: Top: Gaussian curvature $K^{\star}$ versus shear moduli ratio $\alpha$ for a square plate with slenderness $h / L=0.07$ and shear modulus of the bottom layer $G_{b}=10^{7} \mathrm{~Pa}$ (blue line) and $G_{b}=10^{5} \mathrm{~Pa}$ (yellow line). Bottom: Gel configurations at the three points 1-2-3 corresponding to $\alpha=1,0.6,0.3$, respectively, highlighted in the top plot. Colour code corresponds to the volumetric change $J_{d}$, which is related to the amount of solvent uptake.

For $G_{b}=10^{5} \mathrm{~Pa}$ and $G_{b}=10^{7} \mathrm{~Pa}$, figure 1 shows the increase in bending of a plate of slenderness $h / L=0.07$, corresponding to the decrease in $\alpha$ : for $\alpha=1$, the plate is homogeneous and the swelling realizes a homothety with the plate keeping its flat shape. For $\alpha \neq 1$ a sphere-like shape is realized, with equal principal curvatures and the Gaussian curvature $K^{\star}$, evaluated at the center of the middle surface, increasing for $\alpha$ decreasing, corresponding to an increase of the swelling mismatch. We can see that, at $\alpha=0.3$, we get a swelling mismatch $\delta=1.3$ for both the plates, even if, it corresponds to free-swelling stretches $\lambda_{o b}$ and $\lambda_{o t}$ equal to 2.75 and 3.5 , for $G_{b}=10^{5} \mathrm{~Pa}$, and to free-swelling stretches $\lambda_{o b}$ and $\lambda_{o t}$ equal to 1.32 and 1.68 , for $G_{b}=10^{7} \mathrm{~Pa}$. Figure 1 also

\footnotetext{
${ }^{1}$ It has been shown in [27] how for the same values of all the other parameters, $\beta=0.6$ determines the maximum value of curvature in bilayered beams.
} 
shows the plate configurations at the three points 1-2-3 corresponding to $\alpha=1,0.6,0.3$, respectively, highlighted in the plot of figure 1, corresponding to a bottom layer with $G_{b}=10^{7} \mathrm{~Pa}$.

The sphere-like shape still holds when the slenderness $h / L$ changes in the interval $(0.08,0.2)$. Figure 3 shows the final configurations of the plates corresponding to $\alpha=0.7$, that is to a swelling mismatch $\delta \simeq 1$, and to a shear modulus of the bottom layer equal to $G_{b}=10^{5}$. We still get a double-curved surface even if the volumetric change $J_{d}$ is very high as well as the swelling stretches of the two layers.

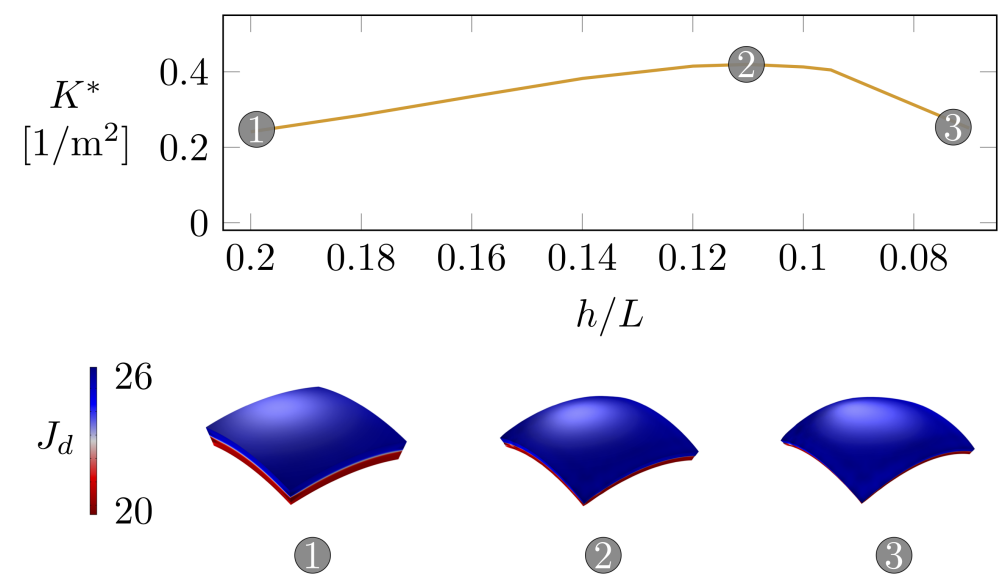

Figure 2: Top: Gaussian curvature $K^{\star}$ versus slenderness $h / L$ for a square plate with slenderness $\alpha=0.7$ and shear modulus of the bottom layer $G_{b}=10^{5} \mathrm{~Pa}$. Bottom: Gel configurations at the three points 1-2-3 corresponding to $\alpha=1,0.5,0.3$, respectively, highlighted in the top plot. Colour code corresponds to the volumetric change $J_{d}$, which is related to the amount of solvent uptake.

\subsection{From spherical to nearly developable shapes}

Within the wide range of swelling tests we investigated, the aspect ratio of the bilayered plate looks like a determinant of the final shape realized by the plate. Indeed, under the same conditions above presented about the materials of the two layers and for a slenderness ratio within the interval explored above, we found a deformation regime completely different. We keep $L_{2}=L$ fixed and changed $L_{1}$ so to cover a range of aspect ratios AR between 1 and 0.1. We runned several simulations with different meshes, for any $\mathrm{AR} \neq 1$, fixing $G_{b}=10^{7} \mathrm{~Pa}, \alpha=0.7$, and $h / L=0.07$. 


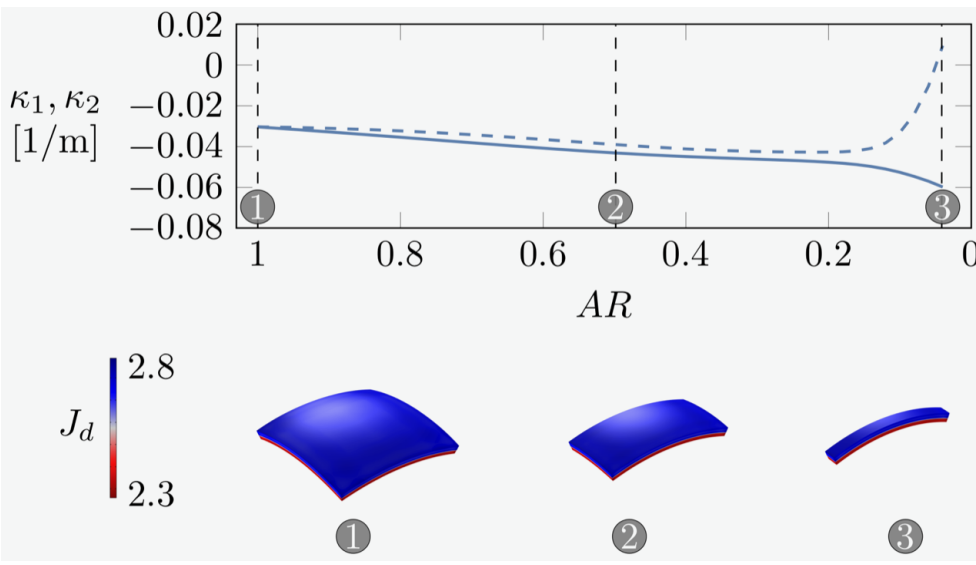

Figure 3: Top: Principal curvatures $\kappa_{1}$ (solid line) and $\kappa_{2}$ (dashed line) versus the aspect ratio AR for a bilayered plate with slenderness $h / L=0.07$, shear modulus of the bottom layer $G_{b}=10^{7} \mathrm{~Pa}$, and $\alpha=0.7$. Bottom: Gel configurations at the three points $1-2-3$ corresponding to $\mathrm{AR}=1,0.5,0.1$, respectively, highlighted in the top plot. Colour code corresponds to the volumetric change $J_{d}$, which is related to the amount of solvent uptake.

The shape of the bilayered plate bifurcates from the spherical shape at AR $=1$, as it is shown in figure 3 where the values of the principal curvatures $\kappa_{1}$ (solid line) and $\kappa_{2}$ (dashed line), evaluated at the center of the middle surface, are represented versus AR. The two lines start diverging when $\mathrm{AR} \neq 1$; they remain close up to a critical AR value (about $\mathrm{AR}=0.2$ in the figure). Figure 3 also shows that a cylindrical shape, with zero Gaussian curvature $K=\kappa_{1} \kappa_{2}$ is attained when AR is very small; otherwise, dome-like shapes with positive Gaussian curvatures are realized.

\subsection{Bifurcation thresholds}

The shaping discussed above can be reviewed within the modeling presented in [34. Therein, the non euclidean plate theory has been used to derive the threshold between the deformative regimes of bilayered plates delivering double-curved and nearly developable shapes, when one of the two layers in-plane grows with respect to the other (in-plane growth mismatch). In that case, the threshold is represented in terms of the natural curvature $\kappa_{o}$ of the plate. Defined as $\bar{\kappa}_{o b}$ the dimensionless natural curvature corresponding to the bifurcation, it is shown that ${ }^{2}$

$$
\bar{\kappa}_{o b}=\left(\frac{20+14 \sqrt{2}}{27}\right)^{1 / 2} \epsilon^{2}, \quad \epsilon=\frac{h}{\mathcal{S}},
$$

with the shape factor $\mathcal{S}=\left(2 / 9 \int r^{4} d A / A\right)^{1 / 4}$ and $\bar{\kappa}_{o b}=\kappa_{o b} h$. At that value of natural curvature, the energy of a spherical cap coincides with the energy of an isometric state, and bifurcation occurs. Taking into account the observations made in [35], where the non

\footnotetext{
${ }^{2}$ See equation (9) in 34 .
} 
euclidean plate theory has been slightly reviewed to comprehend bilayered plate with isotropically growing layers, a revised version of the above formula can be produced, with $\epsilon^{2}$ changed in

$$
\tilde{\epsilon}^{2}=\lambda_{o b} f(\alpha, \beta) \epsilon^{2}, \quad f(\alpha, \beta)=1-\beta+\beta \alpha^{-1 / 5},
$$

and $\beta=h_{t} / h$.

We can easily evaluate $\tilde{\epsilon}$ and, hence, $\kappa_{o b}$ for our plates; we can also evaluate $\kappa_{o}$ cutting a bilayered beam out the bilayered plate and identifying the natural curvature $\kappa_{o}$ with the curvature realized by that beam through the explicit formula produced in [27]. Let us focus on the plates whose steady curved shapes are represented in figure 3 . Fixed $G_{b}$, $\alpha, h / L$ and $L$, the natural curvature $\kappa_{o}$ does not depend on the aspect ratio and, using the equations (4.14)-(4.16) in [27], its value is $69.5 \mathrm{~m}^{-1}$. On the contrary, the bifurcation natural curvature $\kappa_{o b}$ depends on the aspect ratio by means of the shape factor $\mathcal{S}$ and decreases from $55.71 \mathrm{~m}^{-1}$ to $22.85 \mathrm{~m}^{-1}$ going from $\mathrm{AR}=0.1 \mathrm{AR}=1$, as the area of the plate descreases. It means that $\kappa_{o}>\kappa_{o b}$ when $\mathrm{AR} \neq 1$ and, as figure 3 shows, bifurcation from sphere has already occurred. However, $\kappa_{o}>\kappa_{o b}$ also for squared plates which take spherical shapes in the range of swelling tests we investigated.

As we are going to stress in the Conclusions, the poor matching of the two approaches requires further investigation, starting from both sides: numerical tests via a fully threedimensional stress-diffusion model and analytical approach via energetic issues which take into account the characteristics of the swelling deformation processes.

\section{Enforcing cylindrical shaping by anisotropic swelling}

A controlled assembly of isotropic and anisotropic gel layers which admit differential swelling mismatches in different directions greatly changes the shaping scenario; moreover, the architecture of the assembly determines the shaping of the structure. Well-know examples come from plant world. Typically, plant cell walls are composites of stiff cellulose fibrils embedded in a compliant and highly swellable matrix consisting of hemicelluloses. Different alignments of cellulose fibrils in different layers of the composite realize different architectures, and plants can control the swelling(shrinking)-driven deformation of cells by an elaborated adjustment of cell wall architecture [5, 36]. In this section, we start our investigation by the squared and rectangular bilayered isotropic plates above studied and move towards more complex architectures. As first, we consider the assembly of two gel layers with the top softer than the bottom $(\alpha<1)$ and the reinforcing fibers in the $\mathbf{e}_{2}$ direction which are homogeneously distributed in the softer top layer (architecture T), in the harder bottom layer (architecture B), and in both of them (architecture TB). We identify the three architectures with three different marks in the next figures.

We set the swelling problem in the same conditions already introduced in the previous section by considering bilayered plates with $G_{b}=10^{5} \mathrm{~Pa}, \alpha=0.7, \beta=0.6$ and $h / L=0.007$ and different aspect ratios. Then, we realize the three architectures above described; each of them corresponds to a different swelling problem. 
For squared plates, figure 4 (left column) shows that embedding fibers in just one layer favours cylindrical shaping. When the softer top layer is reinforced (top left), stretching in the fiber direction is too expensive and the plate contracts at the top layer so realizing a positive $\kappa_{2}$ curvature as figure 5 shows (see the dashed blue line), with the other curvature $\kappa_{1}$ almost null. On the contrary, when the harder bottom layer is reinforced (middle left), the plate contracts at the bottom layer so realizing a negative $\kappa_{2}$ curvature as figure 5 shows (see the dashed yellow line), again with the other curvature $\kappa_{1}$ almost null. In this case, the steady shape resembles a cylindrical tube as the lower shear modulus of the top layer allows there large stretches in the circumferential (fiber) direction. Two anisotropic layers sharing fiber direction almost do not bend in the fiber direction, as figure 4 (bottom left) shows. Moreover, the anticlastic effect, that is, the difference between the absolute values of the principal curvatures, is reduced with respect to what we observe when only one layer is fiber reinforced. This effect can be appreciated in figure 5 for both $\mathrm{AR}=1$ and $\mathrm{AR}=.6$.

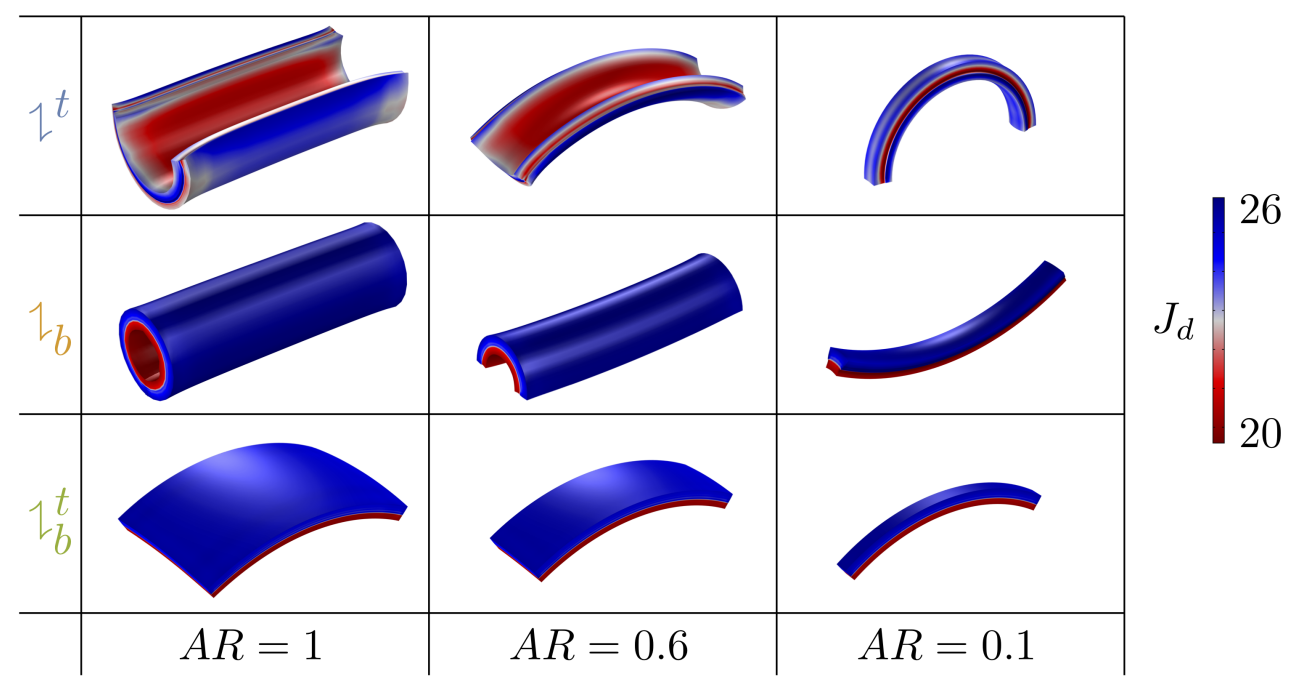

Figure 4: Morphological phase diagrams of emerging surface patterns from numerical simulations corresponding to architectures $\mathrm{T}, \mathrm{B}, \mathrm{TB}$ (top, middle, bottom rows) and to aspect ratios $\mathrm{AR}=1,0.6,0.1$ (left, middle, right columns). Colour code represents the volume change $J_{d}$.

When the aspect ratio is small, plates almost resemble beams. In this case, putting fibers in the softer top or in the harder bottom layer realize shapes with the two principal curvatures of opposite sign, that is, an anticlastic curvature different from zero. Moreover, going from the beam-like plate with the top layer reinforced, figure 4 (top right), to the other, figure 4 (middle right), the signs of both the principal curvatures change. Hence, even in beam-like plates, fibers can greatly affect the steady shape realized under swelling by the structure. 


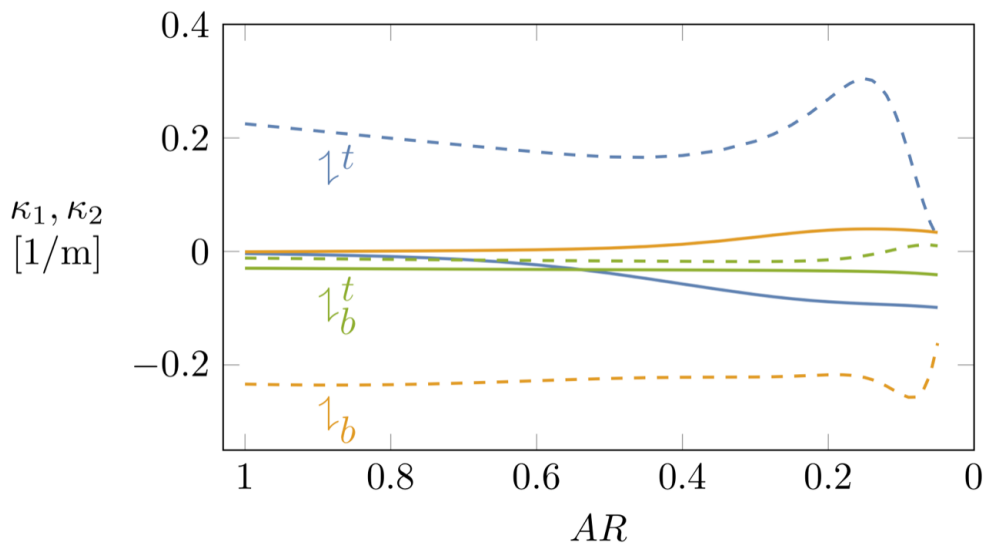

Figure 5: Principal curvatures $\kappa_{1}$ (solid lines) and $\kappa_{2}$ (dashed lines) of bilayered plates assemblied in the architectures T, B, TB (blue, yellow and green lines) versus the aspect ratio AR.

\section{Conclusions}

The behavior of bilayered thin gel sheets under swelling has been investigated using a three-dimensional model which accounts for large deformations and for the contribution of the mixing energy to the total potential energy. Specifically, we focused our attention on the steady shapes realized under swelling and on how these shapes can be mastered. The aspect ratio of the plate has a key role in determining the shape, as already noted for different stimuli-responsive plates in other studies. By contrast, the difference between the elastic properties of the two layers appears to be less important to achieve different shapes. We showed as reinforcing fibers can be crucial to change this last statement. When fibers are inserted just in one layer, we observed a better enforcing of cylinder-like shapes than with fibers in both layers. Finally, a larger suppression of the anticlastic curvature was observed by inserting fibers in both layers.

The results of the wide number of swelling tests here presented set a few challenging issues which have to be considered in the next future, possibly through a semi-analytic approach. It would allow a better control of the role of different quantities here discussed, such as the anisotropic swelling mismatch and the anticlastic effect. Moreover, in our opinion it should also be better discussed the effectiveness of the reduced models of growing plates in predicting final steady shapes when three-dimensional elastic growth (swelling) is involved. As noted in [18, 41, the choice of elastic energies for thin plates and shells is an unsettled issue with consequences for much recent modeling of soft matter.

\section{Acknowledgments}

The work is supported by MIUR (the Italian Ministry of University and Research) through the Project PRIN2017 (N. 2017KL4EF3) Mathematics of active materials from mechanobiology to smart devices. D.B. and M.C. acknowledge the Italian National Group of Mathematical Physics (GNFMNdAM) for support. 


\section{Appendix A. Weak form}

The Finite Elements Model (FEM) solves the balance equations 2.6 and the volumetric constraint $J_{d}=\hat{J}_{d}\left(c_{d}\right)$ in integral form. The problem is reformulated as follows: find $\mathbf{u}_{d}, c_{d}$ and $p$ such that, for all test fields $\tilde{\mathbf{u}}_{d}, \tilde{c}_{d}$, and $\tilde{p}$, it holds

$$
0=\int_{\mathcal{G}_{d}}\left(-\left[\mathbf{S}_{d}\left(\mathbf{F}_{d}\right)-p \mathbf{F}_{d}^{\star}\right] \cdot \nabla \tilde{\mathbf{u}}_{d}+\mathbf{h}_{d} \cdot \nabla \tilde{c}_{d}+\left[J_{d}-\hat{J}_{d}\left(c_{d}\right)\right] \cdot \tilde{p}\right)
$$

where

$$
\mathbf{S}_{d}\left(\mathbf{F}_{d}\right)=G_{d} \mathbf{F}_{d} \quad \text { or } \quad \mathbf{S}_{d}\left(\mathbf{F}_{d}\right)=G_{d} \mathbf{F}_{d}+2 G_{d} \gamma\left(\mathbf{F}_{d} \mathbf{e} \cdot \mathbf{F}_{d} \mathbf{e}-1\right) \mathbf{F}_{d} \mathbf{e} \otimes \mathbf{e}
$$

in the isotropic or anisotropic case, respectively.

Mechanical boundary conditions are easy to handle, as we assume zero tractions and assign a displacement $\overline{\mathbf{u}}_{d}$ on the boundary that eliminates any rigid motion without generating reaction forces. On the contrary, tackling the chemical boundary conditions is more tricky, as it is not possible to control the solvent source $q_{s}$ at the surface, nor the surface concentration $c_{s}$. We set them as

$$
\begin{gathered}
0=\int_{\partial_{c} \mathcal{G}_{d}}\left[\hat{\mu}\left(c_{s}\right)+p \Omega-\mu_{e}\right] \cdot \tilde{c}_{s}, \\
0=\int_{\partial_{c} \mathcal{G}_{d}}\left[\left(c_{d}-c_{s}\right) \tilde{q}_{s}+q_{s}\left(\tilde{c}_{d}-\tilde{c}_{s}\right)\right],
\end{gathered}
$$

and reformulate the complete problem as follows: find $\mathbf{u}_{d}, c_{d}, p, c_{s}$, and $q_{s}$ such that, for any test functions $\tilde{\mathbf{u}}_{d}, \tilde{c}_{d}, \tilde{p}, \tilde{c}_{s}$, and $\tilde{q}_{s}$, equations A.1 A.4 hold; the three fields $\mathbf{u}_{d}, c_{d}, p$ are defined in $\mathcal{G}_{d} \times \mathcal{T}$, while the two fields $c_{s}$ and $q_{s}$ are defined on $\partial_{c} \mathcal{G}_{d} \times \mathcal{T}$.

\section{Appendix B. Numerical implementation and details}

The robustness and the accuracy of the numerical model are tested for a different number $n$ of parallelepiped elements along the three sides of the body, see Fig. B.6. The bilayer plate has the following parameters: $\alpha=0.7, h / L=0.07, \beta=0.6$ and $G_{b}=10^{7}$ Pa.The Gaussian curvature $K^{*}$ at the center of the plate is evaluated and reaches a stable value for $n \geq 5$. The degrees of freedom go from 7000 for $n=2$ to 350000 for $n=8$. In all the simulations $n=6$ parallelepiped elements are used. Since the geometry of the bilayer bodies, investigated in this paper, is always very simple (no curved surfaces), parallelepiped elements are preferred with respect to tetrahedral elements. The convergence of the model is always assured with quintic order Lagrange shape functions for the balance of forces and the balance of solvent mass and a quartic discontinuous Lagrange shape function for the Lagrangian multiplier of the volumetric constraint equation. By contrast, the model does not always converge with lower orders of shape functions, especially for large deformation cases. Moreover, high orders of shape functions are also mandatory to obtain a good accuracy as both the dependent variables $\mathbf{u}_{d}$ and $c_{d}$ in the balance laws have second spatial derivatives. 

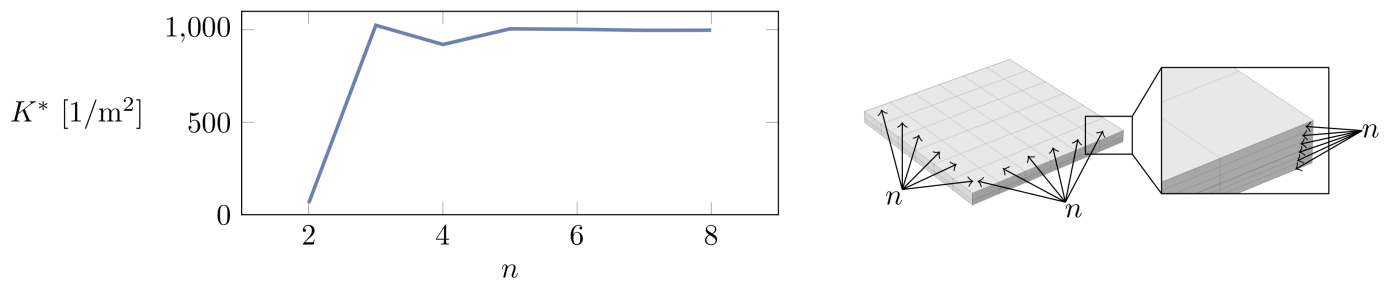

Figure B.6: Validation test for different number of parallelepiped elements $n$ showed at the right of the figure. The Gaussian curvature evaluated at the center of the bilayer body returns stable results for $n \geq 5$.

\section{References}

[1] S. Armon, E. Efrati, R. Kupferman, E. Sharon, Geometry and mechanics in the opening of chiral seed pods, Science 333 (6050) (2011) 17261730. arXiv:http://www.sciencemag.org/content/333/6050/1726.full.pdf, doi:10.1126/science.1203874.

URL http://www . sciencemag.org/content/333/6050/1726.abstract

[2] M. Bacca, O. A. Saleh, R. M. McMeeking, Contraction of polymer gels created by the activity of molecular motors, Soft Matter 15 (2019) 4467-4475. doi:10.1039/ C8SM02598C,

URL http://dx.doi .org/10.1039/C8SM02598C

[3] M. Battista, M. Curatolo, P. Nardinocchi, Swelling-induced eversion and flattening in naturally curved gel beams, International Journal of Mechanical Sciences 161-162, (2019) 105071.

[4] A. Bernheim-Groswasser, N. S. Gov, S. A. Safran, S. Tzlil, Living matter: Mesoscopic active materials, Advanced Materials 30 (41) 1707028. arXiv:https:// onlinelibrary.wiley.com/doi/pdf/10.1002/adma.201707028, doi:10.1002/ adma.201707028.

URL https://onlinelibrary.wiley.com/doi/abs/10.1002/adma.201707028

[5] I. Burgert, P. Fratzl, Actuation systems in plants as prototypes for bioinspired devices, Philosophical Transactions of the Royal Society A: Mathematical, Physical and Engineering Sciences 367 (1893) (2009) 1541-1557. arXiv: https://royalsocietypublishing.org/doi/pdf/10.1098/rsta.2009.0003, doi:10.1098/rsta.2009.0003.

URL https://royalsocietypublishing.org/doi/abs/10.1098/rsta.2009. 0003

[6] A. Cangialosi, C. Yoon, J. Liu, Q. Huang, J. Guo, T. D. Nguyen, D. H. Gracias, R. Schulman, Dna sequence-directed shape change of photopatterned hydrogels via high-degree swelling, Science 357 (6356) (2017) 1126-1130. arXiv:https: //science.sciencemag.org/content/357/6356/1126.full.pdf, doi:10.1126/ 
science.aan3925.

URL https://science.sciencemag.org/content/357/6356/1126

[7] S. A. Chester, L. Anand, A coupled theory of fluid permeation and large deformations for elastomeric materials, Journal of the Mechanics and Physics of Solids 58 (11) (2010) 1879 - 1906. doi:http://dx.doi.org/10.1016/j.jmps.2010.07. 020 .

URL

http://www.sciencedirect.com/science/article/pii/ S0022509610001493

[8] M. Curatolo, S. Gabriele, L. Teresi, Swelling and growth: a constitutive framework for active solids, Meccanica 52 (14) (2017) 3443-3456. doi:10.1007/ s11012-017-0629-x.

URL https://doi.org/10.1007/s11012-017-0629-x

[9] M. Curatolo, P. Nardinocchi, E. Puntel, L. Teresi, Transient instabilities in the swelling dynamics of a hydrogel sphere, Journal of Applied Physics 122 (14) (2017) 145109. arXiv:https://doi.org/10.1063/1.5007229, doi:10.1063/1.5007229. URL https://doi.org/10.1063/1.5007229

[10] M. Curatolo, Effective negative swelling of hydrogel-solid composites, Extreme Mechanics Letters 25 (2018) 46-52.

[11] M. Curatolo, P. Nardinocchi, Swelling-induced bending and pumping in homogeneous thin sheets, Journal of Applied Physics 124 (8) (2018) 085108. arXiv:https: //doi.org/10.1063/1.5043580, doi:10.1063/1.5043580

URL https://doi .org/10.1063/1.5043580

[12] M. Curatolo, P. Nardinocchi, L. Teresi, Driving water cavitation in a hydrogel cavity, Soft Matter 14 (2018) 2310-2321. doi:10.1039/C8SM00100F.

URL http://dx.doi.org/10.1039/C8SM00100F

[13] M. Dias, J. A Hanna, C. Santangelo, Programmed buckling by controlled lateral swelling in a thin elastic sheet, Physical review. E, Statistical, nonlinear, and soft matter physics 84 (2011) 036603. doi:10.1103/PhysRevE.84.036603.

[14] A. I. Egunov, J. G. Korvink, V. A. Luchnikov, Polydimethylsiloxane bilayer films with an embedded spontaneous curvature, Soft Matter 12 (2016) 45-52. doi:10. 1039/C5SM01139F.

URL http://dx.doi .org/10.1039/C5SM01139F

[15] R. M. Erb, J. S. Sander, R. Grisch, A. Studart, Self-shaping composites with programmable bioinspired microstructures, Nat Commun 4 (10) (2013) 1712. doi: $10.1038 /$ ncomms 2666 .

[16] P. J. Flory, J. Rehner, Statistical mechanics of cross-linked polymer networks i. rubberlike elasticity, J Chem Phys 11 (11) (1943) 512-520. doi:10.1063/1.1723791. 
[17] P. J. Flory, J. Rehner, Statistical mechanics of cross-linked polymer networks ii. swelling, J Chem Phys 11 (11) (1943) 521-526. doi:10.1063/1.1723792.

[18] J. A. Hanna, Some observations on variational elasticity and its application to plates and membranes, Zeitschrift für angewandte Mathematik und Physik 70 (3) (2019) 76. doi:10.1007/s00033-019-1122-2. URL https://doi.org/10.1007/s00033-019-1122-2

[19] W. Hong, X. Zhao, J. Zhou, Z. Suo, A theory of coupled diffusion and large deformation in polymeric gels, Journal of the Mechanics and Physics of Solids 56 (5) (2008) 1779 - 1793. doi:http://doi.org/10.1016/j.jmps.2007.11.010. URL http://www.sciencedirect.com/science/article/pii/ S0022509607002244

[20] W. Hong, Z. Liu, Z. Suo, Inhomogeneous swelling of a gel in equilibrium with a solvent and mechanical load, International Journal of Solids and Structures 46 (17) (2009) 3282 -3289. doi:http://dx.doi.org/10.1016/j.ijsolstr.2009.04.022. URL http://www.sciencedirect.com/science/article/pii/ S0020768309001899

[21] H. Huang, A. J. Petruska, M. S. Sakar, M. Skoura, F. Ullrich, Q. Zhang., S. Pané, B. J. Nelson, Self-folding hydrogel bilayer for enhanced drug loading, encapsulation, and transport, in: 2016 38th Annual International Conference of the IEEE Engineering in Medicine and Biology Society (EMBC), 2016, pp. 2103-2106. doi:10.1109/EMBC.2016.7591143.

[22] H. Jiang, L. Fan, S. Yan, F. Li, H. Li, J. Tang, Tough and electro-responsive hydrogel actuators with bidirectional bending behavior, Nanoscale 11 (2019) 22312237. doi:10.1039/C8NR07863G.

URL http://dx.doi .org/10.1039/C8NR07863G

[23] J. Kim, J. A. Hanna, M. Byun, C. D. Santangelo, R. C. Hayward, Designing responsive buckled surfaces by halftone gel lithography, Science 335 (6073) (2012) 1201-1205. doi:10.1126/science.1215309.

[24] J. Kim, C. Kim, Y. Song, S.-G. Jeong, T.-S. Kim, C.-S. Lee, Reversible self-bending soft hydrogel microstructures with mechanically optimized designs, Chemical Engineering Journal 321 (2017) 384 - 393. doi:https://doi.org/10.1016/j.cej.2017.03.125. URL http://wWw.sciencedirect.com/science/article/pii/ S1385894717304928

[25] Y. Liu, J. Genzer, M. D. Dickey, "2d or not 2d": Shape-programming polymer sheets, Progress in Polymer Science 52 (2016) 79 - 106. doi:https: //doi.org/10.1016/j.progpolymsci.2015.09.001. URL http://www.sciencedirect.com/science/article/pii/ S0079670015001021 
[26] A. Lucantonio, P. Nardinocchi, L. Teresi, Transient analysis of swelling-induced large deformations in polymer gels, Journal of the Mechanics and Physics of Solids 61 (1) (2013) 205 - 218. doi:10.1016/j.jmps.2012.07.010.

[27] A. Lucantonio, P. Nardinocchi, M. Pezzulla, Swelling-induced and controlled curving in layered gel beams, Proceedings of the Royal Society A: Mathematical, Physical and Engineering Science 470 (2171). doi:10.1098/rspa.2014.0467.

[28] A. Lucantonio, M. Roche, P. Nardinocchi, H. A. Stone, Buckling dynamics of a solvent-stimulated stretched elastomeric sheet, Soft Matter 10 (2014) 2800-2804. doi:10.1039/C3SM52941J.

URL http://dx.doi .org/10.1039/C3SM52941J

[29] D. Morales, B. Bharti, M. D. Dickey, O. D. Velev, Bending of responsive hydrogel sheets guided by field-assembled microparticle endoskeleton structures, Small 12 (17) 2283-2290. arXiv:https://onlinelibrary.wiley.com/doi/pdf/ 10.1002/smll.201600037, doi:10.1002/smll.201600037. URL https://onlinelibrary.wiley.com/doi/abs/10.1002/smll.201600037

[30] P. Nardinocchi, M. Pezzulla, L. Teresi, Steady and transient analysis of anisotropic swelling in fibered gels, Journal of Applied Physics 118 (24). doi:http://dx.doi. org/10.1063/1.4938737.

URL http://scitation.aip.org/content/aip/journal/jap/118/24/10.1063/ 1.4938737

[31] P. Nardinocchi, M. Pezzulla, L. Teresi, Anisotropic swelling of thin gel sheets, Soft Matter 11 (2015) 1492-1499. doi:10.1039/C4SM02485K.

URL http://dx.doi.org/10.1039/C4SM02485K

[32] K. Oliver, A. Seddon, R. S. Trask, Morphing in nature and beyond: a review of natural and synthetic shape-changing materials and mechanisms, Journal of Materials Science 51 (24) (2016) 10663-10689. doi:10.1007/s10853-016-0295-8. URL https://doi .org/10.1007/s10853-016-0295-8

[33] M. Pezzulla, S. A. Shillig, P. Nardinocchi, D. P. Holmes, Morphing of geometric composites via residual swelling, Soft Matter 11 (2015) 5812-5820. doi:10.1039/ C5SM00863H.

URL http://dx.doi.org/10.1039/C5SM00863H

[34] M. Pezzulla, G. P. Smith, P. Nardinocchi, D. P. Holmes, Geometry and mechanics of thin growing bilayers, Soft Matter 12 (2016) 4435-4442. doi:10.1039/C6SM00246C. URL http://dx.doi .org/10.1039/C6SM00246C

[35] M. Pezzulla, N. Stoop, X. Jiang, D. P. Holmes, Curvature-driven morphing of non-euclidean shells, Proceedings of the Royal Society A: Mathematical, Physical and Engineering Sciences 473 (2201) (2017) 20170087. arXiv: https://royalsocietypublishing.org/doi/pdf/10.1098/rspa.2017.0087, 
doi:10.1098/rspa.2017.0087.

URL https://royalsocietypublishing.org/doi/abs/10.1098/rspa.2017. 0087

[36] M. Ruggeberg, I. Burgert, Bio-inspired wooden actuators for large scale applications, PLoS ONE 10 (2015) e0120718.

[37] G. Stoychev, L. Guiducci, S. Turcaud, J. W. C. Dunlop, L. Ionov, Hole-programmed superfast multistep folding of hydrogel bilayers, Advanced Functional Materials 26 (42) 7733-7739. arXiv:https://onlinelibrary.wiley.com/doi/pdf/10. 1002/adfm.201602394, doi:10.1002/adfm.201602394.

URL https://onlinelibrary.wiley.com/doi/abs/10.1002/adfm. 201602394

[38] T. van Manen, S. Janbaz, A. A. Zadpoor, Programming the shapeshifting of flat soft matter, Materials Today 21 (2) (2018) 144 - 163. doi:https://doi.org/10.1016/j.mattod.2017.08.026

URL http://www.sciencedirect.com/science/article/pii/ S1369702117302237

[39] N. Vu-Bac, T.X. Duong, T.Lahmer, X.Zhuang, R.A.Sauer, H.S.Park, T.Rabczuk, A NURBS-based inverse analysis for reconstruction of nonlinear deformations of thin shell structures, Computer Methods in Applied Mechanics and Engineering 331, (2018) 427-455.

[40] N. Vu-Bac, T.X. Duong, T. Lahmer, P. Areias, R.A. Sauer, H.S. Park, T.Rabczuk, A NURBS-based inverse analysis of thermal expansion induced morphing of thin shells, Computer Methods in Applied Mechanics and Engineering 350, (2019) 480510 .

[41] H. G. Wood, J. A. Hanna, Contrasting bending energies from bulk elastic theories, Soft Matter 15 (2019) 2411-2417. doi:10.1039/C8SM02297F.

URL http://dx.doi .org/10.1039/C8SM02297F

[42] J. Zhang, X. Zhao, Z. Suo, H. Jiang, A finite element method for transient analysis of concurrent large deformation and mass transport in gels, Journal of Applied Physics 105 (9) (2009) 093522. doi:10.1063/1.3106628. 